\title{
Uso de progesterona y sales de estradiol para mejorar la eficiencia reproductiva de vacas con cría *
}

\author{
Paez, P.; Callejas, S. ${ }^{2}$ \\ ${ }^{1}$ Actividad privada, ${ }^{2}$ Área de Reproducción, Facultad de Ciencias Veterinarias, Universidad Nacional del Centro \\ de la Provincia de Buenos Aires (UNCPBA), Paraje Arroyo Seco s/n, Tandil (7000), Buenos Aires, Argentina. \\ Tel.: 02293-439850.E-mail: callejas@vet.unicen.edu.ar.
}

\begin{abstract}
Resumen
Paez, P.; Callejas, S.: Uso de progesterona y sales de estradiol para mejorar la eficiencia reproductiva de vacas con cría. Rev. vet. 21: 2, 140-143, 2010. El objetivo del trabajo fue evaluar la eficiencia reproductiva de un rodeo de vacas con cría ante la administración de un dispositivo intravaginal con $1 \mathrm{~g}$ de progesterona (DISP) más una inyección de benzoato de estradiol (BE) combinado o no con la administración de cipionato de estradiol (CPE) al retirar el DISP. Se utilizaron 196 vacas Angus negro de primera parición (condición corporal: 2 a 2,5 - escala 1 a 5), post parto $\geq$ a 90 días y mayoritariamente en anestro. Las vacas fueron divididas al azar para recibir tres tratamientos: 1) Grupo BEDISP $(\mathrm{n}=65)$ : el día 0 recibieron un DISP más $2 \mathrm{mg}$ de BE permaneciendo colocado 8 días. 2) Grupo BEDISPCPE ( $\mathrm{n}=65$ ): ídem al grupo BEDISP sumando una inyección de CPE (1 mg) al retirar el DISP. 3) Grupo control ( $\mathrm{n}=66)$ : no recibieron tratamiento hormonal. El servicio comenzó el día 8, utilizando un 4\% de toros Angus (duración: 90 días). El diagnóstico de gestación se realizó por ultrasonografía, a los 59 días de retirado los DISP para determinar el porcentaje de preñez en los primeros 21 días de servicio y a los 50 días del retiro de los toros para determinar la preñez final. Se evaluaron los efectos de los tratamientos sobre el porcentaje de preñez en 21 días de servicio, final y distribución de la preñez. El grupo BEDISPCPE tuvo el mayor porcentaje de preñez en los primeros 21 días de servicio (BEDISP: 36,9\%, BEDISPCPE: 53,8\% y control: $36,4 \%, p<0,05)$, no difiriendo la preñez final $(91,3 \%$; $p>0,05)$; además tuvo el mayor porcentaje de preñez cabeza. Se concluye que el uso de un DISP combinado con una inyección de $\mathrm{BE}$ al momento de colocarlo y una de $\mathrm{CPE}$ al momento de retirarlo, mejoran la eficiencia reproductiva de vacas con cría que están mayoritariamente en anestro.
\end{abstract}

Palabras clave: vaca de cría, inducción actividad sexual, progesterona, cipionato de estradiol.

\begin{abstract}
Paez, P.; Callejas, S.: Use of progesterone and estradiol salts to improve reproductive efficiency of beef cows. Rev. Vet. 21: 2, 140-143, 2010. The objective of this work was to evaluate the effect of a progesterone intravaginal device with $1 \mathrm{~g}$ of progesterone (IVD) plus estradiol benzoate injection (EB), combined or not with the administration of estradiol cypionate (ECP) at device removal upon reproductive efficiency. One hundred and ninety-six first calving Angus cows were used (body condition: 2 to 2.5 - scale 1-5), post partum $\geq 90$ days and mostly in anestrous. The cows were randomly assigned to receive three treatments: 1) EBIVD group $(n=65)$ : On day 0 IVD was fitted and EB injected, IVD remaining placed 8 days. 2) EBIVDECP $(\mathrm{n}=65)$ : idem EBIVD group by adding an injection of ECP $(1 \mathrm{mg})$ at IVD removal. 3) Control group $(n=66)$ : not receiving hormone treatment. The breeding service began on day 8 , using $4 \%$ of Angus bulls (duration: 90 days). The pregnancy diagnosis was made by ultrasonography, 59 days after IVD removal to evaluate the pregnancy rate on the first 21 days of breeding service and 50 days of retired bulls to evaluate the final pregnancy. The effects of treatments on the pregnancy rates within 21 days of breeding service, as well as all service and pregnancy distribution were evaluated. EBIVDECP group had the highest pregnancy rate in the first 21 days of breeding service (EBIVD: 36.9\%, EBIVDECP: $53.8 \%$ and control: $36.4 \%, \mathrm{p}<0.05)$. However, no differences were observed in the final pregnancy rate (91.3\%), but distribution of pregnancies along the breeding period was different,
\end{abstract}

\footnotetext{
* Parte del Proyecto "Aumento de la eficiencia de técnicas aplicadas a la reproducción en rumiantes" (03/H218 - UNCPBA). Recibido: 31 agosto 2010 / Aceptado: 14 octubre 2010
} 
since head pregnancies accounted for the highest percentage of total pregnancies. In conclusion, the use of IVD combined with an injection of EB at the time of its insertion and ECP during device removal improves reproductive efficiency of suckled cow that are in anestrous.

Key words: beef cows, activity sexual induction, progesterone, estradiol cypionate.

\section{INTRODUCCIÓN}

El uso de dispositivos intravaginales con progesterona combinado con sales de estrógenos, administradas al momento de colocar y retirar el dispositivo, sumado a una inyección de un agente luteolítico al final del tratamiento, ha sido utilizado para controlar el ciclo estral e implementar programas de inseminación artificial a tiempo fijo ${ }^{2}$. Este tratamiento permite sincronizar la ovulación de los animales que se encuentran ciclando, así como inducir actividad en aquéllos que están en anestro.

El fundamento de inducir actividad sexual en vacas en anestro mediante el uso de dispositivos intravaginales con progesterona se basa en que al permanecer los mismos colocados durante $7 \mathrm{u} 8$ días se generan niveles de progesterona en sangre que imitan la primera fase luteal que se produce luego de ocurrido el parto, la cual es de corta duración en la mayoría de los casos ${ }^{10}$. A posteriori de ésta, los animales comienzan a tener ciclos estrales regulares.

En base a lo planteado precedentemente, se realizó un trabajo con la finalidad de inducir actividad sexual en vacas con cría que recibirían servicio natural. Así, se ha informado un aumento en el porcentaje de preñez en los primeros días de un servicio natural en aquellas vacas tratadas con un dispositivo con $1 \mathrm{~g}$ de progesterona y que recibieron benzoato de estradiol (BE) al momento de colocar el dispositivo intravaginal. En este tratamiento no se administró el agente luteolítico dado que la mayoría de las vacas estaba en anestro, como así tampoco se inyectó el BE final ${ }^{3}$.

En otros trabajos se utilizaron dispositivos con menos de $1 \mathrm{~g}$ de progesterona y se llegó a la conclusión que para que el dispositivo tuviera un efecto mejorador sobre el porcentaje de preñez, era necesario no solo administrar BE inicial, sino también se debía administrar $\mathrm{BE}$ al momento de retirar el dispositivo ${ }^{9}$ o 24 horas después ${ }^{4}$.

Luego de analizar los resultados obtenidos con dispositivos con menos de $1 \mathrm{~g}$ de progesterona, surgió el interés de evaluar los efectos de la administración de estrógenos al finalizar un tratamiento de inducción de actividad sexual utilizando dispositivos con $1 \mathrm{~g}$ de progesterona; en consecuencia, el objetivo del presente trabajo fue evaluar el efecto de utilizar un dispositivo intravaginal con $1 \mathrm{~g}$ de progesterona más una inyección de BE al momento de colocarlo, combinado o no con la administración de cipionato de estradiol al retirar el dispositivo, sobre la eficiencia reproductiva de un rodeo de vacas con cría al pie.

\section{MATERIAL Y MÉTODOS}

Lugar, animales, alimentación. El trabajo se realizó en el establecimiento agropecuario "Los Pirineos", Provincia de Buenos Aires (Argentina), el cual posee una superficie total de 2.100 ha y se corresponde a la región geomorfológica conocida como pampa deprimida.

Se utilizaron 196 vacas con cría Aberdeen Angus negro de primera parición, las cuales habían recibido su primer servicio a los 20-22 meses de edad, con un rango de condición corporal de 2 a 2,5 (escala 1 a 5) y un post parto mayor o igual a 75 días. Cabe señalar que en el año en que se realizó el trabajo (2009), hubo periodos de lluvias con registros inferiores a los normales, lo cual condicionó la oferta forrajera y esto se tradujo en una pobre condición corporal de los vientres al inicio del periodo de servicio. Quince días antes de comenzar con los tratamientos, el rodeo fue revisado ginecológicamente por palpación transrrectal determinando que el $76,5 \%$ de las vacas presentaba ovarios sin estructuras palpables y un útero fláccido; la mayoría del resto de las vacas tenía folículos de 8 a $10 \mathrm{~mm}$ de diámetro y muy pocas poseían cuerpos lúteos (menos del $10 \%$ del rodeo).

La alimentación de los animales se basó en promociones de verdeos de invierno y pastizales naturales de buena calidad, destacándose que a partir del examen ginecológico las vacas tuvieron acceso a un lote de verdeo de invierno con muy buena disponibilidad forrajera. Los vientres mostraron ganancia de peso, traducida en una mejoría de su condición corporal, durante todo el periodo del servicio y luego de finalizado el mismo. El rango de condición corporal al momento de realizar el diagnóstico de gestación fue de 3 a 3,5.

Tratamientos y servicio. Las vacas fueron divididas al azar en tres grupos, para recibir los siguientes tratamientos: Grupo BEDISP $(\mathrm{n}=65)$ : el día 0 recibieron un dispositivo intravaginal con progesterona (DIB, Syntex) más una inyección intramuscular de $2 \mathrm{mg}$ de benzoato de estradiol (Syntex). El dispositivo permaneció colocado durante 8 días. Grupo BEDISPCPE $(n=65)$ : ídem al grupo BEDISP con la salvedad que se administró $1 \mathrm{mg}$ de cipionato de estradiol CPE (Cypiosin, Syntex) intramuscular, en el momento de retirar el dispositivo. Grupo control $(n=66)$ : no recibió tratamiento hormonal. El servicio natural comenzó el día 8 y tuvo una duración de 90 días; se utilizó un 4\% de toros Aberdeen Angus, el 50\% de ellos con alta capacidad de servicio y el resto de media capacidad de ser- 
vicio ${ }^{1}$. Los toros tenían una edad de 2 a 3 años, siendo negativos a tritrichomoniasis y campylobacteriosis.

Diagnóstico de gestación. El diagnóstico de preñez se realizó por medio de ultrasonografía (Falco Vet, Esaote-Pie Medical, Holanda), a los 59 días de retirado los dispositivos para determinar el porcentaje de vacas preñadas en los primeros 21 días de servicio y a los 50 días de retirados los toros para determinar la preñez final.

Análisis estadístico. Se estudió el efecto de los tratamientos sobre las tasas de preñez en los primeros 21 días de servicio y final, como así también sobre la distribución de preñez (grande y chica). Se consideró preñez grande a aquella que se obtuvo en los primeros 60 días de servicio y preñez chica a la ocurrida en los últimos 30 días. Para los cálculos estadísticos, se utilizó el Proc. CATMOD del paquete SAS, fijando un nivel de confianza del $95 \%(\alpha=0,05)$.

\section{RESULTADOS}

Se observó un efecto significativo del tratamiento sobre el porcentaje de preñez en los primeros 21 días de servicio $(p<0,05)$. Las vacas que fueron tratadas con un dispositivo intravaginal con progesterona y recibieron una inyección de CPE al momento de retirarlo, se preñaron en mayor proporción que aquéllas que no recibieron la sal de estradiol o ningún tratamiento hormonal ( $\mathrm{p}<0,05$; Tabla 1). La preñez final no difirió entre tratamientos $(91,3 \% ; \mathrm{p}>0,05)$.

Los animales que recibieron el tratamiento con progesterona y CPE tuvieron una mayor proporción de vacas con preñez grande comparada con el grupo control $(\mathrm{p}<0,05$; Tabla 2$)$ y tendieron a diferir del grupo $\operatorname{BEDISP}(p=0,09)$.

\section{DISCUSIÓN}

El uso de dispositivos intravaginales con $1 \mathrm{~g}$ de progesterona en vacas en anestro y con servicio natural ha mostrado ser efectivo para inducir actividad sexual; así, se ha informado de un aumento significativo $(p<0,05)$ en el porcentaje de vacas preñadas en los primeros 15 días de servicio a partir de colocado el dispositivo (28,3\% vs. $18,0 \%$; vacas que recibieron o no el tratamiento con progesterona) ${ }^{5}$.

En otro trabajo, en el cual se realizaron dos ensayos y se utilizó además del dispositivo una inyección de benzoato de estradiol al momento de colocarlo, el porcentaje de preñez fue significativamente superior para el grupo tratado $(33,9 \%)$ comparado con el grupo control $(10,8 \%)^{3}$; en este ensayo el periodo de servicio varió de 17 a 23 días. En coincidencia con estos trabajos, se ha informado de una mejoría en los primeros 7 días de servicio ${ }^{7}$, utilizando un protocolo anteriormente descrito ${ }^{3}$ y en un trabajo publicado recientemente, el $63,9 \%$ de las vacas que recibieron el tratamiento hor-
Tabla 1. Tasas de preñez en los primeros 21 días de servicio.

\begin{tabular}{lc}
\hline tratamiento & preñez $(\%)$ \\
\hline BEDISP & $36,9^{\mathrm{a}}(24 / 65)$ \\
BEDISPCPE & $53,8^{\mathrm{b}}(35 / 65)$ \\
control & $36,4^{\mathrm{a}}(24 / 66)$ \\
\hline
\end{tabular}

BEDISP: $1 \mathrm{~g}$ progesterona $+2 \mathrm{mg}$ benzoato de estradiol al colocar el dispositivo; BEDISPCPE: ídem BEDISP + $1 \mathrm{mg}$ de cipionato de estradiol al retiro del dispositivo. ${ }^{\mathrm{a}, \mathrm{b}}$ Valores con superíndices diferentes difieren $\mathrm{p}<0,05$.

Tabla 2. Porcentaje de vacas con preñez grande en 90 días de servicio.

\begin{tabular}{lc}
\hline tratamiento & preñez grande $(\%)$ \\
\hline BEDISP & $68,4^{\text {ay }}(39 / 57)$ \\
BEDISPCPE & $82,0^{\text {bx }}(50 / 61)$ \\
control & $62,3^{\text {a }}(38 / 61)$ \\
\hline
\end{tabular}

BEDISP: $1 \mathrm{~g}$ de progesterona $+2 \mathrm{mg}$ de benzoato de estradiol al colocar el dispositivo; BEDISPCPE: ídem BEDISP $+1 \mathrm{mg}$ de cipionato de estradiol al retiro del dispositivo. Valores con superíndices diferentes difieren a,b: $\mathrm{p}<0,05 \mathrm{o}$ tienden a diferir $\mathrm{x}, \mathrm{y}: \mathrm{p}<0,10$.

monal se preñó en los primeros 19 días de servicio en comparación con el 35,6\% del grupo control ${ }^{8}$.

En el presente trabajo, el grupo de vacas que recibió el dispositivo con progesterona y $\mathrm{BE}$ inyectado al momento de colocarlo no tuvo un mayor porcentaje de preñez comparado con el grupo control, discrepando con los resultados informados previamente. Por el contrario, el uso del CPE administrado en el momento de retirar el dispositivo produjo una mejoría en la eficiencia reproductiva, traducida en mayor porcentaje de preñez en los primeros 21 días de servicio y mayor porcentaje de vacas preñadas en los primeros 60 días de servicio. Este resultado coincide con lo informado por varios investigadores que han utilizado dispositivos que tienen menos de $1 \mathrm{~g}$ de progesterona. Así, en 20 días de servicio no observaron una mejoría en el porcentaje de preñez en vacas que recibieron un dispositivo con $0,5 \mathrm{~g}$ de progesterona más BE al momento de colocarlo (35\%) comparadas con aquéllas que no recibieron tratamiento hormonal $(40 \%)^{6}$. Por el contrario, cuando a los dispositivos con menos de $1 \mathrm{~g}$ de progesterona $(0,558 \mathrm{~g})$ se les adicionó $\mathrm{BE}$ al momento de retirarlo ${ }^{4} \mathrm{o}$ a las 24 horas posteriores ${ }^{9}$ se mejoró significativamente la eficiencia reproductiva, cuando se compara con la no administración de la sal de estrógeno o vacas que no recibieron tratamiento hormonal. Un comportamiento similar fue observado en el presente trabajo; no obstante, los dispositivos utilizados contenían $1 \mathrm{~g}$ de progesterona.

La mejoría observada en el porcentaje de preñez en los primeros 21 días de servicio en el grupo BEDISPCPE permitió que una mayor proporción de animales quedaran preñados en los primeros 60 días de servicio. En consecuencia, los animales parirán antes y tendrán 
mayores probabilidades de preñarse en forma anticipada en la próxima temporada de servicio. Esto constituye un beneficio adicional a la mejora que se produciría en la producción de $\mathrm{kg}$ de terneros destetados por haberse preñado en forma anticipada.

En síntesis, se ha observado que el uso de sales de estradiol al final del tratamiento con un dispositivo que contiene menos de $1 \mathrm{~g}$ de progesterona para inducir actividad sexual en vacas con servicio natural, sería necesario para mejorar la eficiencia reproductiva; por el contrario, esto no siempre ha sucedido con dispositivos que tienen $1 \mathrm{~g}$. No obstante, siempre y cuando no se muestre un efecto perjudicial de la sal de estradiol administrada al finalizar el tratamiento con $1 \mathrm{~g}$ de progesterona, sería conveniente su administración en función de los resultados obtenidos en el presente trabajo. Futuras investigaciones deberán confirmar los resultados aquí informados.

Se concluye que el uso de un dispositivo intravaginal con $1 \mathrm{~g}$ de progesterona combinado con una inyección de benzoato de estradiol al momento de colocarlo y una de cipionato de estradiol al momento de retirarlo, mejoran la eficiencia reproductiva de vacas con cría que se encuentran mayoritariamente en anestro.

\section{REFERENCIAS}

1. Acuña CM. 1992. Capacidad de servicio en toros: análisis del período 1985-1992. Vet Arg 9: 488-495.

2. Callejas S. 2005. Control farmacológico del ciclo estral bovino: bases fisiológicas, protocolos y resultados. Parte II. Rev Taurus 25: 16-35.

3. Callejas S, Alvarez S, Zarzaso M, Cledou G. 2007. Uso de un dispositivo intravaginal con progesterona en vacas de cría con servicio natural. Anales VII Simposio Internacional de Reproducción Animal IRAC, Córdoba (Argentina), p. 236.
4. Callejas S, Schang S, Cledou G, Cesio T. 2009. Uso de dispositivos intravaginales con progesterona combinados con benzoato de estradiol al retiro en vacas con cría en servicio natural. Anales VIII Simposio Internacional de Reproducción Animal. IRAC, Córdoba (Argentina), CD: bovinos $/ 8$.

5. Colombo M, DeVillafañe P, Cledou G, Bartolomé J. 2007. Administración de un dispositivo intravaginal de progesterona en vacas en anestro durante el servicio natural para adelantar la concepción. Anales VII Simposio Internacional de Reproducción Animal IRAC, Córdoba (Argentina), p. 299.

6. Echevarria S, Zapiola A, Callejas S. 2009. Uso de dispositivos con $0,5 \mathrm{~g}$ de progesterona en vacas con cría cola de parición con servicio natural. Anales VIII Simposio Internacional de Reproducción Animal IRAC, Córdoba. (Argentina), CD: bovinos/22.

7. González Chaves S, Uslenghi G, Cledou G, Cabodevila J, Callejas S. 2009. Efecto de la utilización de dispositivos intravaginales con progesterona sobre el porcentaje de preñez en vacas con servicio natural que recibieron o no una IATF. Anales VIII Simposio Internacional de Reproducción Animal IRAC, Córdoba (Argentina), CD: bovi$\operatorname{nos} / 25$.

8. González Chaves S, Cledou G, Cabodevila J, Callejas S. 2010. Uso de dispositivos intravaginales con progesterona e IATF para aumentar la eficiencia reproductiva en un rodeo de cría. Memorias XXXVIII Jornadas Uruguayas de Buiatría, Paysandú (Uruguay), p. 110-111.

9. Grigera J, Cledou G, Callejas S. 2009. Uso de dispositivos intravaginales con $0,558 \mathrm{~g}$ de progesterona combinado con benzoato de estradiol en vacas con cría en servicio natural. Anales VIII Simposio Internacional de Reproducción Animal IRAC, Córdoba (Argentina). CD: bovinos/30.

10. Murphy MG, Boland MP, Roche JE. 1990. Pattern of follicular growth and resumption of ovarian activity in postpartum beef sucker cows. J Reprod Fert 90: 523-533. 